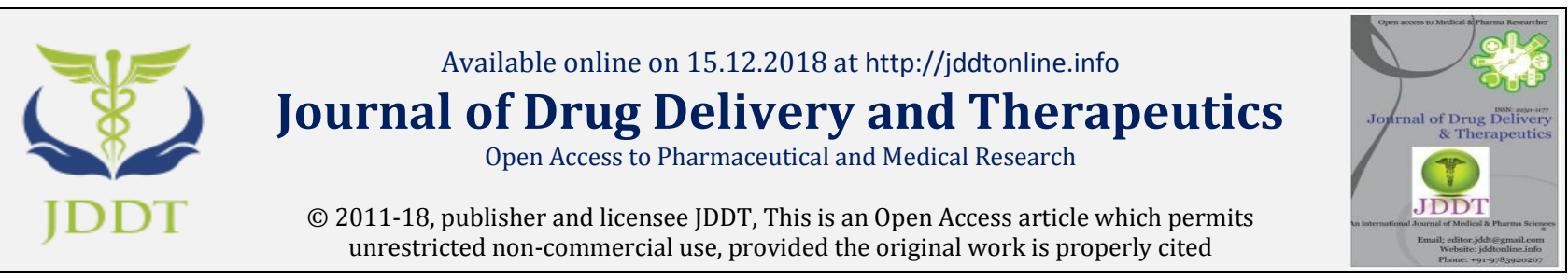

Open $\odot$ Access

Review Article

\title{
Computer system validation in the perspective of the pharmaceutical industry
}

\author{
${ }^{1}$ Singh Asheesh*, ${ }^{1}$ Singour Pradeep ${ }^{2}$ Singh Parul \\ ${ }^{1}$ Faculty of Pharmacy, VNS institute, Neelbud Bhopal (M.P.), India- 462044 \\ ${ }^{2}$ Department of Pharmacy, Guru Ghasidas University, Bilaspur (C.G.), India - 495009
}

\begin{abstract}
Computer Systems Validation (CSV) is a process used to ensure (and document) that a computer based systems will produce information or data that meet a set of defined requirements. If a system meets these requirements, it can be assumed that it is consistently performing in the way it was intended. Quality is an imperative for customers whenever they consider a product or service. It is also important as it relates to life-saving products such as pharmaceuticals. In this regard, the Food and Drug Administration introduced good manufacturing practice (GMP) to maintain and improve the quality of pharmaceutical products. GMP ensures that products are consistently produced and controlled according to the quality standards appropriate to the intended use and as required by the marketing authorization. One of the major GMP requirements is that all of the critical manufacturing equipment, utilities, and facilities in the pharmaceutical industries must be properly qualified and validated prior to production. Currently, this practice forms the core of the regulations that are strictly followed by pharmaceutical companies worldwide. A validation assessment program is a necessity in the pharma industry to ensure adherence to pharmaceutical cGMP guidelines, and to help companies maintain consistent quality. The same principles are applied in computer system validation to a computer system or an information technology system. It's essential to maintain quality standards in pharma since nonconformance can have far-reaching consequences. Computer system validation checks the effectiveness and the efficiency with which the system is meeting the purpose for which it was designed. This study aims to identify needs of computer system validation of instrument/equipment practiced in the perspective of pharmaceutical industry.
\end{abstract}

Keywords: Computer system validation, Validation, Qualification, GAMP

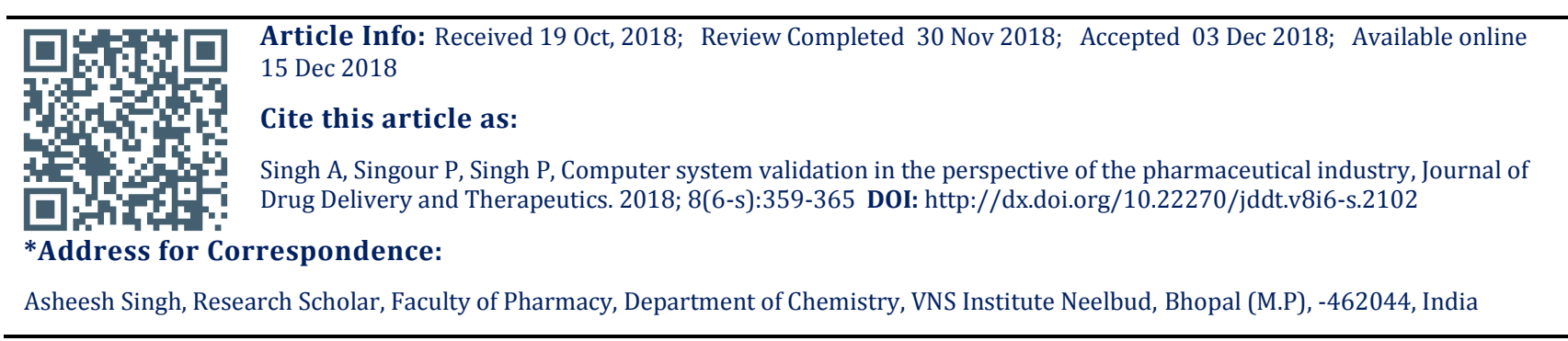

\section{INTRODUCTION}

The concept of validation was first proposed by Ted Byers and Bud Loftus in the mid-1970s to improve the quality of pharmaceutical products 1. Currently, in the pharmaceutical manufacturing industry, validation plays a vital role in producing high-quality pharmaceutical products that meet good manufacturing practice (GMP) guidelines. Validation is an important requirement imposed by authorities worldwide to regulate the production of pharmaceutical and medical devices. An equipment, utility, or facility that is not validated may produce inferior outputs 2. Thus, the Food and Drug Administration (FDA) requires validation, which is defined as the process of collecting and evaluating data to draw scientific evidence that an equipment, utility, or facility is capable of consistently delivering quality products. On the one hand, validation involves confirmation by examination and provision of objective evidence that the particular requirements for a specific intended use are fulfilled ${ }^{3}$. In the pharmaceutical concept, validation refers to the establishment of documented evidence that an equipment, utility, or system, when operated within established parameters, can perform effectively in producing a medicinal product that meets the predetermined specifications. Validation of software and computer systems follows the same principle as the qualification of instrument hardware. USP $<1058>$ has a short chapter on software validation. Software is divided into three categories

1. Firmware integrated as chips into instrument hardware for control through local user interface.

2. Software for instrument control, data acquisition, and data processing. An example would be a chromatography data system.

3. Standalone software, for example a Laboratory Information Management System (LIMS) package.

Most valuable is the statement about firmware: "Firmware is considered as a component of hardware of the instrument itself. Indeed the qualification of hardware is not possible without operating its firmware. Thus when the hardware is qualified at the user's site, the integrated 
firmware is also essentially qualified. No separate on-site qualification of the firmware is needed."4

\section{NEED OF VALIDATION, QUALIFICATION AND IT SYSTEM VALIDATION}

Pharmaceutical facilities consist of various processes, each of which must be accurate to ensure that the end product is of high quality. While validation is concerned mainly with processes, it is referred to as a qualification when the same approach is applied to a machine or equipment instead of a process $^{5}$.

Validation is a systematic approach where it is confirmed that any process in a pharmaceutical facility will operate within the specified parameters whenever required. This is achieved by collecting and analyzing data. Validation is done to assure that the processes will produce consistent and repeatable results within the predetermined specifications. Validation is needed as it verifies whether the quality standards and compliance are being met by the product in real time, which is really important in every pharmaceutical facility. Further, it also establishes that the facility is meeting current good manufacturing practice (cGMP) guidelines that are set for the industry by concerned regulatory bodies. Validation can be considered as a documented evidence of the process meeting the predetermined specifications.

No pharmaceutical plant is complete without an IT system, which is responsible for controlling, supporting and documenting various processes. It is extremely important to validate the computer and IT systems as it makes sure that all the concerned IT applications are fulfilling their intended purposes. Validation helps in controlling different phases of development, design, testing and routine of the software that is being used by the IT system during its life cycle. As long as the computer system is running accurately, you can be assured that all the information and reports that they store remain safe. You must implement stringent quality requirements in GMP-regulated industries to control the procedures throughout the Software Development Life Cycle (SDLC). Focus the validation efforts on crucial aspects such as risk analysis and in-depth validation approach. Make sure that you apply the documentation to the computerized system as it manages crucial data that has an impact on the quality of the products. The components of computer system validation include all the activities that are involved in applying the appropriate controls throughout the SDLC and for procedures that are necessary for creating the documentation ${ }^{6-8}$.

\section{ANALYTICAL INSTRUMENT QUALIFICATION \& IMPORTANT OF CALIBRATION}

Before you conduct validation, you must complete the process of qualification. It is a systematic process that starts by the project phases of the installations, equipment and utilities. Analytical Instrumentation Qualification, also known as AIQ, is the documented process where a complex and sophisticated measurement device is demonstrated to be accurate, precise and selective enough for the intended analytical measurement. This is carried out to determine the sustainability and qualification of any instrument for the intended purpose. Qualification is not limited to a validation process, but it is a part of it. It can be further divided into installation qualification (IQ), operation qualification (OQ) or performance qualification (PQ). Based on the operation and function of equipment, system or utility, you must make installation qualification and operation necessary. They should be monitored and calibrated periodically and they must be submitted to preventive maintenance. If you want to fulfill the GMP requirements for quality assurance of products, you must consider several factors such as infrastructure, equipments and raw materials. Make sure that the whole production process is controlled until the final product is released. Since all the three processes, validation, calibration and qualification, are extremely critical in pharmaceutical processes, you must ensure that you have the right outsourcing partner to conduct them. When you have the correct partner by your side, you get vital assistance in maintaining GMP standards, while keeping the costs down. Look for important traits while choosing a partner, such as their quality management system, their ability meet all your requirements while containing the costs, response to fluctuating workloads, etc. Make sure you tie up with the partner who is right for your company, and there will be one less thing that you have to worry about.

To make sure that validation of processes is legitimate, you have to do calibration. It is a process which demonstrates that an instrument is producing results within the specified limits as compared to those produced by a traceable standard over an appropriate range of measurements. It is extremely crucial that all calibration activities are performed with qualified instruments by an accredited laboratory. You must ensure that you always use calibrated instruments in manufacturing. Using uncalibrated instruments can lead to safety risks, which is an absolute no-no for any pharmaceutical company. You will see an increase in waste if you use uncalibrated instruments. Further, equipment of poor quality will cost you a lot of money too, putting a financial burden on your company. Calibrate your equipment periodically to ensure that the measurements and outputs achieved are accurate at all times without affecting the quality of the final product.9-11

\section{GOOD AUTOMATED MANUFACTURING PRACTICE (GAMP)}

GAMP stands for Good Automated Manufacturing Practice. Usually when one hears the terms GAMP, it is in reference to a guidance document entitled GAMP-5: A Risk-Based Approach to Compliant GxP Computerized Systems. GAMP-5's approach can be summed up by the V-model diagram in the beginning, or at least in GAMP 4, there were five categories of software:

\section{- Category 1: Operating systems \\ - Category 2: Firmware \\ - Category 3: Standard software \\ - Category 4: Configured software \\ - Category 5: Custom software}

There was always a debate about some commercial software packages were they category 3 or 4? Many spectroscopists would argue that an application should be classified as category 3 and not 4 , as it should be less work to validate and evade the real classification. To help resolve this debate, in GAMP 5 the software categories have been revised and refined most for the better and one for the worse. This is a natural evolution of this approach to software classification. So, we now have the following four categories:

- Category 1: Infrastructure Software

- Category 3: Nonconfigured products

- Category 4: Configured products

- Category 5: Custom applications 
Why Classify Software: Before we go into a detailed discussion of the software categories, perhaps we should ask the question "Why bother to classify software?". What benefit does this software classification provide? If you look at Table I there is a built-in risk assessment. The least risky and most widely available software is in category 1 (operating systems, databases, office software, and other widely available software). This is widely available software that can be used by anyone and in any industry. As we progress through down the categories as shown in Table I, generally the software becomes more specialized in its function (from a general office application to software that can control a spectrometer to acquire and process data then report the results). As we go down the list there is the increasing ability of the users to change the operation of software and process the results until we reach category 5 . In category 5 is a unique solution that is conceived, specified, written, tested, and maintained by the users or the organization; here is the greatest risk. Let's now take a detailed look at each of the software categories and see what has changed and if there are any problems we need to discuss.

Software Classification Changes and Their Laboratory Impact: Presented and discussed here are the various changes to the software classifications in the new GAMP guide.

Category 1: Greatly Expanded Scope-Infrastructure Software: Category 1 has undergone a radical change and expansion from simply operating systems, that had been constant in GAMP versions 1 to 4 , to infrastructure software. This category is broken down into two subcategories:

- Established or commercially available layered software and

\section{- Infrastructure software tools.}

The intention is that the infrastructure software in this category provides the computing environment for running both regulated and nonregulated applications within an organization. All software in this category needs to be controlled and qualified in an organization so that dual standards are not applied by the IT department, which can question the status of validated applications if not done. Software in the subcategory of Established or Commercially Available Layered Software still includes operating systems from GAMP 4, but this has also been expanded to encompass a greater scope:

- databases

- $\quad$ programming languages

- middleware

- office software

- $\quad$ ladder logic interpreters (for manufacturing systems),

- Statistical programming tools and spreadsheet packages.

The key issue is that many of these software tools are the base products for the applications used in the laboratory or they are the foundation layer for the laboratory applications to operate under. For example, if your spectrometer has application software that has a database to manage the methods, data, and results you generate, the latter is configured by the spectrometer supplier from the out-of-the-box database to operate with their application software. Languages are used as a means to write the applications, each of which will be validated for their intended use. Note that category 1 also includes office software such as word processing, spreadsheet, database, and presentation applications. Now before you rush off thinking that Excel templates and macros do not need to be validated, think again, as the guide notes that "applications developed using these packages" are excluded from category 1 and these can be category 3,4 , or 5 , respectively, depending on their complexity. Note also the phrasing of the subcategory "established or commercially available". This means that both open source and software commercial can be used, which ratifies the status quo (open source operating systems [Linux or OpenVMS], databases [MySQL], and source code management [Sub Version]). In many IT departments and research groups open source software is used and often this use can be extensive. Some people may argue that open source software is hacked code, but when the code can be reviewed by many programmers it may be argued that the quality of the finished application could be better than some commercially available software. Regardless of the debate, the word established allows the use of open source applications within category 1 .

Software Categories 3, 4, and 5: A Quick Reprise: GAMP version 5 has defined these three software categories as follows:

- Category 3: Nonconfigured Products: Off-the-shelf products that cannot be changed to match the business processes but this category also can include configurable software products but where only the default configuration is used.

- Category 4: Configured Products: Configured software products provide standard interfaces and functions that enable configuration of the application to meet user-specific business processes. However, configuration using a vendor-supplied scripting language should be handled as custom components (category 5).

- Category 5: Custom Applications: These applications are developed to meet the specific needs of a regulated company. This definition implicitly includes spreadsheet macros written using visual basic for applications (VBA) and LIMS language customizations. It also will include macros written for some spectroscopy software as short cuts for performing a series of tasks. Note that this is the highest risk software, as there is the greatest likelihood of functional omissions, bugs, and errors in the software, and therefore, the life cycle model used needs to have sufficient controls to ensure that it is properly specified, designed, built, and tested before release.

GAMP 5 notes that these categories are not silos of software but a continuum: There might be elements of a higher or lower category depending how the software is used and/or configured or customized.

Importance of System Life Cycle Model: A software application or a computerized system does not suddenly materialize out of thin air. Each one needs to be planned and implemented. Therefore, the use of a system life cycle is important as it provides a plan to use as a basis for the implementation or building of a computerized system. Note the words:

- Plan: A plan is a blue print or road map for carrying out a task. It will outline the stages or phases that need to 
be gone through so that the system will be built correctly and function as required.

- Basis: Plans can be changed to fit the system that is being implemented or developed and you should accept that there is no one-size-fits-all approach. A specific system can have more, the same, or fewer needs than the standard life cycle model will provide and therefore, adaptation of the plan might be needed to accommodate them.

Therefore, the key requirement of any life cycle model used is that it should be meaningful and applicable to the system that you are building or implementing. If it is not, then you have problems.

CSV is interlinked very closely with the software development life cycle (SDLC) considering that it is conducted at every stage of SDLC. CSV forms part of the testing process with one key differentiation of validation at each step, instead of a single final stage testing. The CSV process is in fact created keeping in view the steps of the SDLC, and is roughly modelled as per an archaic model of the same. The entire process of computer system validation is created keeping in mind the FDA guidelines that have been defined for pharma, in order to meet the quality standards and adhere to the pharmaceutical cGMP. So in a lot of ways, it is the FDA guidelines which have created the framework for CSV and continue to define the way it's shaped and remodeled over time, depending on the dynamic factors in the pharmaceutical industry ${ }^{12-13}$.

\section{PROCESSES NEEDED FOR COMPUTER SYSTEM VALIDATION}

Qualification activities should be described in a master plan. The plan documents a company's approach for specific activities, for example, how to qualify analytical instruments, how to assess vendors or what to test for commercial computer systems. A master plan serves two purposes: when implemented right, it ensures consistent and efficient implementation of equipment qualifications, and it answers an inspector's question for a company's approach for instrument qualification and system validation. A validation master plan is also officially required by Annex 15 to the European GMP directive: "All validation activities should be planned. The key elements of a validation program should be clearly defined and documented in a Validation Master Plan (VMP) or equivalent documents". FDA regulations and guidelines do not specifically require a validation master plan. However, inspectors want to know what the company's approach towards validation is. The qualification master plan is an ideal tool to communicate this approach both internally and to inspectors. In case there are any questions as to why things have been done or not done, the master plan should provide the answers. CSV is dependent on the complexity of the project and can be largely broken down into the following processes:

Master Plan: This checks whether the specifications are in line with user requirements. During this stage, teams are also established which will run the entire process. The set of activities to be carried out during validation are established too. This is basically the process of preparing the blueprint for the entire CSV. This process is the pivot of a validation program since it covers the complete setup such as the physical hardware, software, sites and also validates processes such as risk mitigation and redundancy strategies. Equipment and computer validation master plans should include:
1. Introduction with a scope of the plan, e.g., sites, systems, processes

2. Responsibilities, e.g., user departments, QA, IT

3. Related documents, e.g., risk master plan

4. Products/processes to be validated and/or qualified

5. Qualification/validation approach

6. Risk assessment

7. Steps for equipment qualification and computer system validation with examples on type and extent of testing

8. Vendor assessment

9. Handling existing systems

10. Change Control procedures and templates

11. Instrument obsolescence and removal

12. Training plans (system operation, GMP)

13. Templates and references to SOPs

14. Glossary

Project Plan: The project plan outlines what is to be done in order to get a specific system into compliance. For inspectors, it is a first indication of the control a laboratory has over a specific instrument or system and it also gives a first impression of the qualification quality. This process defines the standard operating procedures for each process in a validation assessment program and is a subset of the master validation plan. More importantly it defines a deadline within which the CSV must be completed. A detailed documentation and training on the standard operating procedures (SOPs) is carried out during this process. Additionally activities such as risk assessment, backup planning and change management are also undertaken during this phase. If a master plan is defining the outline, then a project plan is the execution stage.

Figure 1 shows the link between the master plan and project plan. Ideally master plans are developed at a corporate level. Project plans are written in departments specifically for an instrument or system. Depending on the size, structure and geographic distribution there also may be a site or country specific master plan that is derived from the corporate master plan but has been customized according to specific circumstances and requirements of that site.



Figure 1: Link between master plan and project plan. 
Design Qualification: "Design qualification (DQ) is the documented collection of activities that define the functional and operational specifications of the instrument and criteria for selection of the vendor, based on the intended purpose of the instrument. Design qualification is a shared responsibility between the vendor and the user of an instrument.

Requirement specifications: Requirement specifications of software and computer systems should be linked to test cases in some kind of traceability matrix. This can be a table on its own or the link can be built into the requirements table. Each specification should have a unique ID code. Criticality of the function can be defined as high, medium or low. Most important questions to ask are: what happens if the function does not work at all or if it produces wrong results? In the next column the test priority is documented. Criticality plays a major role but also the question as to how the user's environment or the user, for example through a wrong user configuration, can influence correct functioning.

Installation Qualification (IQ): This stage delves deeper into the installation process and creates checks and balances for any new component that may have been purchased (such as new IT equipment) or any new software or hardware that may have been installed. Tasks performed for IQ include:

- $\quad$ Prepare the laboratory facility according to vendor environmental specifications.

- Control and record environmental conditions, if critical. For example, temperature and humidity.

- Compare equipment received with the purchase order (including, accessories and spare parts).

- Check equipment for any damage.

- Verify that the instrument conforms with physical and construction requirements, as specified by the user.

- Check documentation for completeness (operating manuals, maintenance instructions, standard operating procedures for testing, safety and validation certificates).

- Install hardware (instrument, fittings and tubing for fluid connections, columns in HPLC and GC, power cables, data flow and instrument control cables).

- Switch on the instruments and ensure that all modules power up and perform an electronic self-test.

- List equipment manuals and SOPs.

- Record firmware revision.

- Prepare an installation report.

- Enter instrument data into an inventory data base.

- Prepare, review and sign formal IQ documentation.

Operational Qualification (OQ): “Operational qualification $(O Q)$ is the documented collection of activities necessary to demonstrate that an instrument will function according to its operational specification in the selected environment "Emphasis should be placed on "in the selected environment". Testing of instrument hardware at the user's site is required because instrument characteristics can change when shipped from the vendor to the user, for example through mechanical vibration. The most frequently asked questions related to OQ testing are: what should be tested, which are the acceptance criteria, and who should perform the tests? USP answers all the questions in a single sentence: "Users, or their qualified designees, should perform these tests to verify that the instrument meets manufacturer or user specifications in the user's environment. Designees could be, for example, vendor representatives."
The frequency of $\mathrm{OQ}$ depends on the type of instrument, on the stability of the performance characteristics, but also on the specified acceptance criteria. In general, the time intervals should be selected so that the probability is high that all parameters are still within the operational specifications. Otherwise, analytical results obtained with that particular instrument are questionable. Here the importance of proper selection of the procedures and acceptance limits becomes very apparent. For example, if the baseline noise of a UV/Visible detector is set to the lowest possible limit as specified by the vendor, the lamp will have to be changed more frequently than when set at a factor of 5 higher.

Performance/Process Qualification (PQ): PQ tests specific applications and proactively engages in maintenance and conducts performance tests. All the processes contribute to effectively meeting pharmaceutical cGMP and help ensure that all the technical systems involved are contributing to help meet the required product quality standards.

Configuration Management and Change Control: The purpose of configuration management is to be aware of the lifetime composition of the system from planning to retirement. The initial or baseline configuration of a system has been documented as part of IQ. Any changes to specifications, programming codes or the initial set up of computer hardware should follow written change control procedures and be documented. Changes may be initiated because errors have been found in the program or because additional or different software functions or hardware may be desirable. Requests for changes should be submitted by users and authorized by the user's supervisor or department manager 14-15.

21 CFR Part 11 - FDA's Regulation on Electronic Records and Signatures: In 1997 the United States Food and Drug Administration (FDA) issued a regulation that provides criteria for acceptance by the FDA of electronic records, electronic signatures and handwritten signatures21. With this regulation, entitled Rule 21 CFR Part 11, electronic records can be equivalent to paper records and handwritten signatures. The rule applies to all industry segments regulated by the FDA that includes Good Laboratory Practice (GLP), Good Clinical Practice (GCP) and current Good Manufacturing Practice (cGMP). Part 11 requires computer systems used in FDA regulated environments to be validated. Chapter 10 (a) states: Computer systems should be validated to ensure accuracy, reliability and consistent intended performance. There is no further instruction on how computer systems should be validated 16-17.

Validation Report: At the end of validation, a summary report should be developed. This should be a mirror of the validation project plan. It should be organized in such a way that it has all the elements and follows the outline of the validation plan. This makes it easy to check if all plan items have been completed successfully. Deviations should be documented, if there are any, together with corrective actions and/or work around solutions. The report should include a statement that the instrument or system is qualified or validated. After the statement and the report have been signed by management, the product can be released for operation. Typically, the validation plan and the report are the first documents inspectors want to see when they inspect a validation project. If everything is well organized and documented, it may well be that after looking at both documents inspectors get such a good 
impression about the validation work that they will focus on other inspection areas.

\section{COMMON COMPUTER SYSTEM VALIDATION PROBLEMS}

Computer System Validation (CSV) in the life sciences was focused on software validation and infrastructure and computing platform qualification for systems that supported FDA-regulated activities and records. Today, organizations are increasingly focusing on overall, global IT compliance, to satisfy 21 CFR Part 11 but also equivalent laws in other countries, Sarbanes-Oxley (SOx), HIPAA, export and shipping regulations, and much more. To meet these varied and global needs, pharmaceutical manufacturers must:

Challenge I. Standards: Various standards exist across the organization. Policies, procedures, work instructions, and templates vary by business, department, or site. Significant costs result from overlapping SOPs and inconsistent standards, which make sharing of assets difficult. Industrywide standard methodologies, guidelines, and tools have been issued by global organizations, such as ISPE and ICH, but in order to make the assets applicable to a wide range of companies, processes, systems, and products, they did not replace the more detailed, localized standards.

Challenge II. Interpretation: A significant cost to validation projects is caused by long debates among the various authors and reviewers, rework, and inconsistent interpretation of standards and requirements. Most regulations include very high-level statements that set objectives, but don't specify how to implement the controls or how much is good enough. For example, 21 CFR Part 11 (11.10 (d)) requires "Limiting system access to authorized individuals." This one statement can be expanded to varied requirements for technical security controls and procedural controls for managing access.

Challenge III. Organization and Governance: Many companies still have decentralized governance and uncontrolled execution. The ownership and management of validation activities vary from project to project and from one department to another. Projects are not handled consistently with clear roles and responsibilities. Some are led by IT, other by users or quality.

Challenge IV. Efficiency Across Sites and Departments: Site-to-site efficiencies have not been achieved due to siteand department-specific procedures, templates, and interpretation. We've seen many cases where multiple sites develop complete validation packages for the same system that they use the same way, because there is no sharing of inventory and project information.

Challenge V. Execution: As stated earlier, we see excessive rework being done by validation teams. Most often, the rework is a result of different opinions and styles of project team members and inconsistent quality of work that is done by unqualified individuals. A common scenario is that individuals perform work that doesn't align with their level and skills. Junior quality reviewers, who are qualified to review documents and identify incomplete or inaccurate information and deviations from standards, end up determining the course of action to remediate the problems. The solution is often redoing the work. On the other hand, we see highly-qualified, expensive resources performing low-level mechanical tasks, because a fixed team is assigned to the project and must share the workload.
Challenge VI. Tools: System life cycle assets, such as templates, outlines, forms, and guidance documents are often inconsistent across departments and are not targeted to drive value. They are put in place to minimize the risk of project team members taking shortcuts and skipping sections, but are not flexible and drive unnecessary efforts with minimal value to quality or compliance. We've seen cases where an extensive validation package was prepared for a new CD writer, or complete detailed Installation Qualification protocol, scripts, and report were produced for the installation of utilities such as WinZip and virus scan.

Challenge VII. Training: Training is usually conducted within each business on standards and processes; however, there is minimal coaching and guidance. The short training that is usually provided is rarely enough to qualify individuals without coaching and support until they gain hands-on experience. Often multiple training sessions have to be taken in a very short period of time, where the individual's ability to absorb, understand and retain the materials is in question.

Challenge VIII. Personnel: Working with many life science companies shows that usually there are capable, knowledgeable central validation groups, but weaker decentralized execution groups. CSV standards are often deployed without the appropriate training and coaching and without assurance of consistent interpretation. Organizations believe that simply reading standard operating procedures (SOPs) and receiving a few hours of training enable individuals to follow a consistent approach ${ }^{18-23}$.

\section{COMMON FDA OBSERVATIONS THAT CAN BE AVOIDED WITH GOOD CSV PRACTICES}

Failure to Validate a Regulated System: Validation activities and the documentation generated during these activities are often an area of interest for inspectors. In the case of Computer System Validation, an inspector would typically look for documented evidence that validation activities were performed in accordance with approved validation procedures. Additionally, the documentation presented to the inspectors should show that applicable regulatory requirements were met.

Absence of Written Procedures: Procedures provide an established, approved framework for carrying out tasks within a regulated environment. And they are often among the first documents requested by inspectors. Validation procedures should document with sufficient detail how validation activities are performed and what deliverables are generated via these activities. The approach to validation taken should also be specified. Ensure that the approach takes into account applicable regulations (such as 21 CFR Part 11) and outlines the methodology used (for example, a risk-based approach following GAMP 5). Moreover, the process stakeholders should be identified and their respective roles and responsibilities should be clearly defined.

Written Procedures Not Followed: When performing computer system validation, make sure that any deviations to the validation plan are documented and a rationale for the deviation is provided. Validation non-conformances should be addressed by identifying the root cause and implementing appropriate corrective actions. The system must not be released for use prior to completing validation and any system limitations should be specified in writing. Changes made to the system once it is validated should be 
put in place in accordance with an approved change request.

Failure to Maintain Records: Computer systems are used to manage a variety of regulated documents, ranging from training records and complaint files to raw data obtained during laboratory and production activities. Failure to demonstrate during validation that a regulated computer system functions as intended can lead to serious problems and potential regulatory observations down the line. Data integrity and security could be compromised, resulting in the inability to produce documentation requested during inspections. If a computer system is used to track reporting to regulatory bodies, such as the mandatory reporting of medical device issues or adverse drug experiences, it is crucial to ensure that the system performs as expected to be sure that reporting deadlines are respected ${ }^{24-29}$.

\section{CONCLUSION}

Pharmaceutical manufacturers can definitely improve their validation projects by performing several measures to minimize or eliminate the deficiencies in the computer system validation problems discussed above. Collaboration, prioritization, planning, oversight, and clarity of purpose can also substantially promote the

\section{REREFENCES}

1. Agalloco, J, Carleton FJ, Validation of Pharmaceutical Process, 3rd Edition, USA, New York: Informa Healthcare, 2008.

2. Sangeetha NKD, Bala krishna P, Development and validation of a UPLC method for the determination of duloxetine hydrochloride residues on pharmaceutical manufacturing equipment surfaces, Pharmaceutical Methods, 2011; 2 (3):161166.

3. Giridhar A, Hamdan I, Joglekar G, Venkatasubramanian V, Reklaitis GV, Real-time process management in particulate and pharmaceutical systems, Computer Aided Chemical Engineering, 2011; 29:1035-1039.

4. Bedson P, Sargent M, The development and application of guidance on equipment qualification of analytical instruments, Accreditation and Quality Assurance, 1996; 1(6):265-274.

5. Coombes P, Laboratory Systems Validation Testing and Practice, DHI Publishing, LTD, Raleigh, USA 2002.

6. US Food \& Drug Administration - Guidance for Industry Quality Systems Approach to Pharmaceutical Current Good Manufacturing Practice Regulations, September 2006.

7. US Food \& Drug Administration - Guidance for Industry Process Validation: General Principles and Practices - Revision 1, January 2011.

8. US Food \& Drug Administration - General Principles of Software Validation; Final Guidance for Industry and FDA Staff, January 2002.

9. US Food \& Drug Administration - Guidance for Industry: 21 CFR Part 11 - Electronic Records and Electronic Signatures: Scope and Application, August 2003.

10. PIC/S Good Practices for computerized systems in regulated "GxP" environment, Pharmaceutical Inspection Co-operation Scheme guidance, September 2007.

11. GAMP Forum - GAMP Guide, A Risk-Based Approach to complaint GxP Computerized Systems - Ver. 5.0

12. GAMP Forum - GAMP Good Practice Guide, Testing of GxP Systems.

13. GAMP Forum - GAMP Good Practice Guide, A Risk-Based Approach to Compliant Electronic Records and Signatures. success of validation projects. Research study on the existing validation frameworks should be carried out to identify possible positive elements which may help to eliminate most of the pitfalls discussed in this paper. These elements can be incorporated in a framework which fulfills the basic framework design requirements. It must be simple, systematic, can easily be understood by the future implementers and flexible enough to adapt itself to different contexts. This framework must be validated by applying it in case studies which must be carried out in pharmaceutical companies to confirm its flexibility, robustness and validity. In order to achieve it, it is suggested that these case studies should be conducted in three different backgrounds in a pharmaceutical company to validate facilities, utility systems and equipments. The possible application of this new validation framework into the existing validation procedure can help future implementers to achieve remarkable improvements in validation scope, thus significantly saving manufacturers' time, effort, and money invested in validation projects.

\section{ACKNOWLEDGEMENT}

Authors are thankful to VNS Institute of Pharmacy, Bhopal and Department of Pharmacy, GGU Bilaspur for providing the necessary facilities \& guidance to complete this review.

14. GAMP Forum - GAMP Good Practice Guide, Global Information Systems Control and Compliance.

15. GAMP Forum - GAMP Good Practice Guide, A Risk-Based Approach to Operation of GxP Computerized Systems - 2010.

16. FDA, "Guidance for Industry: Regulatory Submissions in Electronic Format for New Drug Application (NDA)," http://www.fda.gov/cder/guidance/2353fnl.pdf.

17. FDA, "Guidance for Preparing Data for Electronic ANDA Submission," http://www.fda.gov/cder/ guidance/3223fnl.htm.

18. FDA, "Revised Guidance for Providing Regulatory Submissions to CBER in Electronic Format," http://www.fda.gov/cber/gdlns/ebla.pdf.

19. FDA, "Enforcement Policy: 21 CFR Part 11, Electronic Records, Electronic Signatures” (CPG 7153.17, 13 May 1999), http://www.fda.gov/ ora/compliance_ref/cpg/cpggenl/cpg160-850.htm.

20. FDA, "Public Docket Index for 21 CFR Part 11 (E-Rec/E-Sig Rule)," http://www.fda.gov /ora/compliance_ref/Part1 1/dockets_index.htm.

21. FDAMA, SEC. 410, "Mutual Recognition Agreements and Global Harmonization," http://www.fda.gov /cder/guidance/105115.htm\#SEC.

22. CDER Warning Letters Web site: http://www.fda.gov/foi/warning_letters/m5056n.pdf and http://www.fda.gov/foi/warning_letters/m5057n.pdf.

23. FDA Cyber Warning Letters Web site: http://www.fda.gov/cder/warn/cyber/cyber2017.htm.

24. GAMP and ISPE Web site: http://www.gamp.org and http://www.ispe.org.

25. PDA Web site: http://www.pda.org.

26. PIC Web site: http://www.picscheme.org/index.htm.

27. Information Society Standardization System Web site: http://www.cenorm.be/isss/

28. International Biometric Industry Association Web site: http://www.ibia.org.

29. ComputerValidation.com Web site: http://www.computervalidation.com 